\title{
Personal Counseling on Smart Phones For Smoking Cessation
}

\section{Jeni Paay}

Dept. Computer Science

Aalborg University

Denmark

jeni@cs.aau.dk

Jesper Kjeldskov

Dept. Computer Science

Aalborg University

Denmark

jesper@cs.aau.dk

\section{Mikael B. Skov}

Dept. Computer Science

Aalborg University

Denmark

dubois@cs.aau.dk

Permission to make digital or hard copies of part or all of this work for personal or classroom use is granted without fee provided that copies are not made or distributed for profit or commercial advantage and that

copies bear this notice and the full citation on the first page. Copyrights for third-party components of this work must be honored. For all other uses, contact the Owner/Author.

Copyright is held by the owner/author(s).

CHI'15 Extended Abstracts, Apr 18-23, 2015, Seoul, Republic of Korea ACM 978-1-4503-3146-3/15/04

http://dx.doi.org/10.1145/2702613.2732847

\begin{abstract}
The unhealthy consequences of smoking cigarettes are well known and yet people still continue to smoke. Recent research involving technology to help people quit smoking has had limited success. Personal face-toface counseling has historically proven the most successful and effective means to help people quit, but most people are reluctant or too busy to attend counseling sessions. As a potential solution to this problem, we explore providing personal counseling to users via their mobile phones. The advice, written by experts, is based on data about the user and their actual smoking habits collected through smart phones. From a prototype deployment with users in real life context, we found that this type of personal counseling is influential in changing peoples' smoking behaviors for the better. In addition, being made aware of actual smoking habits helps people form strategies that improve their ability to quit.
\end{abstract}

\section{Author Keywords}

Smoking cessation; health behavior change; mobile apps; personal counseling; self-tracking; interaction design

\section{ACM Classification Keywords}

H.5.m. Information interfaces and presentation (e.g. HCI): Miscellaneous. 


\section{Introduction}

Unhealthy behaviors can cause illness. Smoking cigarettes comes with many diseases such as cancer, lung disease, heart disease, and poor blood circulation [7]. According to the World Health Organization, nearly six million people die each year from smoking-related diseases [11]. Illness and deaths from smoking are not only tragic but also influence the economy within social welfare systems. As a result of this, smoking cessation organizations worldwide put a lot of effort into fighting the smoking of tobacco. However, despite these efforts, the global consumption of tobacco is still growing [11].

For several years $\mathrm{CHI}$ research has studied and tried to influence behavior change within different health domains, such as physical activity, healthy eating and drinking, stress management, etc. This research aims at strengthening the foundation for changing health behavior using technology, but also shows that much more work is needed before we can expect to see real benefits in terms of improved health in society. Although this research has produced positive results, very little has been done in the area of using interactive technologies to facilitate smoking cessation. Personal smart phones represent an interesting platform for reaching people at the times and places where they are tempted to smoke. Our work explores the potential for giving smokers trying to quit access to personal counseling on their smart phones through a prototype application, QuittyLink, which uses health behavior change strategies to assist people trying to quit.

\section{Related Work}

There are limited research examples of technology supporting smoking cessation. Early studies, such as Schumann et al. [8], have used computer-tailored health letters to support participants' smoking cessation, with positive outcomes. Prior to this, Strecher [10] confirmed the efficiency of computertailored content through the web, as opposed to nontailored content. Among the studies exploring the use of mobile technology to support smoking cessation, "Txt2stop" by Free et al. [4] found that sending helpful text messages to participants doubled their quit rate in the short term. Similarly, "Distract Me" by Ploderer et al. [6] showed that distractions and tips on mobile phones could keep users from smoking. Extending on these findings, Paay et al. [5] explored how different types of content sent to mobile phones could help smokers quit. They found that content that relates directly to people's smoking habits, were most helpful.

Abroms et al. [1] reviewed 98 Apps for Android and iPhone, finding that nearly half of them simply provided calculations based on estimates by the user, and that the Apps generally had low adherence to evidencebased practices. The problem with basing feedback on estimates provided by the user with rather long intervals is that data is often incomplete, imprecise and prone to error. To avoid this problem, studies in other health behavior domains have successfully explored the use of tracking technologies for automated and more accurate data input, e.g., "Playful Bottle" [2] monitors the amount of water office workers drink, and "UbiFit" [3] tracks the user's physical activity.

\section{The "QuittyLink" App}

Based on findings from previous studies on health behavior change and smoking cessation we designed and built the "QuittyLink" prototype. QuittyLink is a smoking cessation App designed to add a combination of self- and system- tracking with personal counseling 


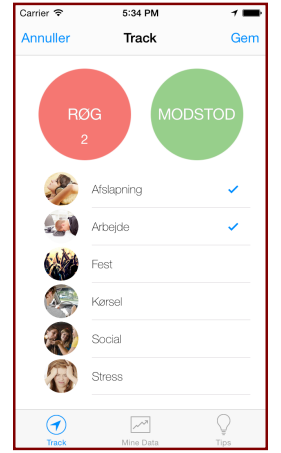

Figure 1.

Track screen.

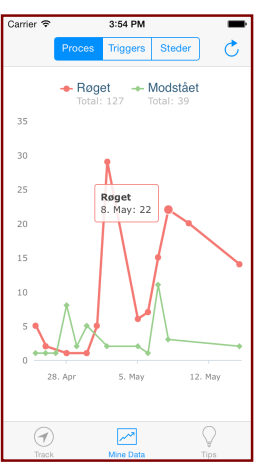

Figure 2.

My Data:

Process

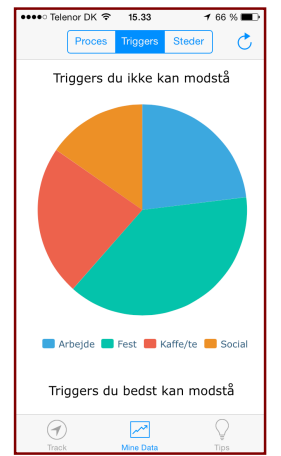

Figure 3.

My Data:

Triggers to help people to quit smoking and stay quit. QuittyLink has native applications for both iOS and Android.

Contextual attributes like time, place and situation can become conditioned cues to smoke. Certain situations trigger strong cravings, for example, working, drinking coffee or dealing with stress. Keeping track of these triggers and other contextual attributes can increase smokers awareness of their behavioral patterns and assist them in making and maintaining strategies to change. To do this, the number of smoked cigarettes, resisted cigarettes and triggering situation are entered by the users at the times these events occur. In addition to this, the QuittyLink system logs the time and location of these entries. By tracking smoked and resisted cigarettes precisely, users become more aware of their smoking patterns. This makes QuittyLink useful to smokers who are thinking of quitting, who are trying to quit, as well as smokers who are working to stay quit. In doing this, we counteract one of the biggest limitations of current apps, that is, most are only useful for smokers who have already quit [1].

Counseling has proven to be highly effective in increasing people's success in quitting smoking [9]. Even so, many people are reluctant to seek it, or attend smoking cessation groups because they see quitting as a private matter, or find it inconvenient to meet at a set time and location [10]. As counseling is the most effective smoking cessation technique [10], we wanted to leverage the privacy and flexibility provided by mobile technologies to deliver regular personal counseling to the smoker. To achieve this, QuittyLink logs data about the user's smoking habits, which is then sent to a Quitline expert. The expert then delivers weekly advice tailored to the individual, which can be read at a place and time of the user's choosing, even reviewed later, potentially reaching smokers who would otherwise not seek personal counseling.

\section{QuittyLink Interaction Design}

Interaction with QuittyLink was kept as simple as possible to encourage people to use it frequently during their day. QuittyLink has three tabs corresponding to the main interactive parts of the app: Track, My Data and Tips. The Track screen (Figure 1) is used to input the number of cigarettes smoked (red button) or resisted (green button) each time. Users can choose from a predefined list of 10 different situations that triggered them to want to smoke. They can tick as many as are relevant or the "other" option if their particular trigger is not listed. The time, date and location of the smoking episode are recorded automatically. All data is saved to a remote server.

On the My Data screen users can select between three different representations of the user's smoking history. The intention is to provoke self-awareness by providing accurate information about a persons smoking habits over time. The Process line graph (Figure 2) shows the number of cigarettes smoked over time (red line) against the number resisted (green line). The Triggers pie chart (Figure 3 ) shows the proportions of different situations that have triggered smoking episodes, for example, work, party, drinking coffee/tea, socializing. The Places bar chart (Figure 4) shows how many cigarettes have been smoked in different locations, given as street addresses. This gives users an understanding of where and when they tend to smoke the most, helping them to reflect on the kinds of locations and situations they need to avoid, or be more aware in resisting their cigarette cravings. 


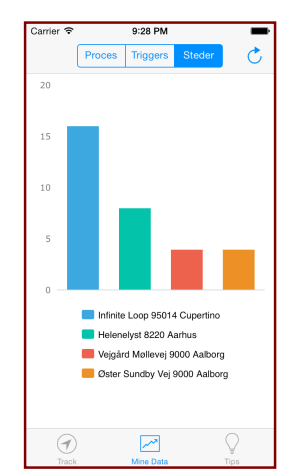

Figure 4.

My Data:

Places

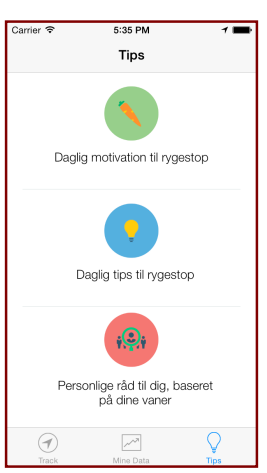

Figure 5.

Tips menu

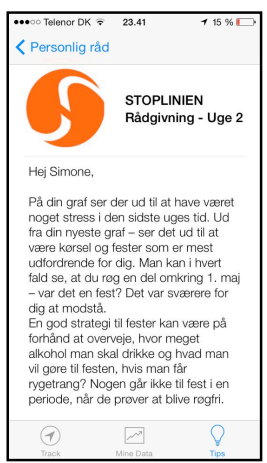

Figure 6.

Personal

counseling
The Tips screen (Figure 5) gives access to three different content types: Daily Motivations (motivational stories), Daily Tips (tips on ways to stop smoking) and Personal Counseling (tailored expert counseling). Motivational stories and daily tips are sourced from quit experts and updated daily. Counseling is provided weekly (Figure 6), as tailored advice fitting the participant's own situation, with the opportunity to contact the counselor directly for follow up advice. A daily SMS reminder was sent to inform users of new content and prompt them to use QuittyLink. Push notifications alerted users to the arrival of their personal counseling messages. All Tips are saved in the App so users can return to them.

\section{Field Study}

We conducted a field study of the QuittyLink App in use in real world settings over a period of 3 weeks. The aim of the field study was to explore how smokers responded to entering data about their smoking habits to receive personal counseling advice based on their input. We also wanted to see if the My Data visualizations prompted self-reflection on their smoking habits, as well as the effect of this and the counseling on their ability to quit. The field study was conducted in Denmark in spring of 2014. The App was delivered using public App stores. All participants were asked to use the App every day for the duration of the study.
Participants completed a demographic survey, which included information about their smoking habits. There were 13 participants in the study, 4 males and 9 females. Their ages ranged from 22 to 52 years old. Seven of the participants had an iPhone and six had an Android device. Eleven participants had previously tried to quit smoking. Six were thinking about quitting, 4 were preparing to quit and 3 were trying to stay quit. Based on Fagerströms test of nicotine dependency, 3 participants had no dependency, 2 had very low dependence, 6 participants had low to moderate dependence and 2 had high nicotine dependence.

During the study, each participant was asked to use the App every day whenever he/she smoked a cigarette or had a craving. Once a week, the tracked data for each participant was collected and sent to the counselor. Based on their initial survey responses and the tracked data from the past week, participants then received personal counseling messages, typically 15-25 lines, from the expert at Stoplinien counseling service.

After the study, a semi-structured interview, approx. 45 minutes long, was conducted with each of the participants to discuss their experience with the App and the role it played in their smoking cessation.

Questions covered general impressions of the system, entering data, the graphs, their counseling messages and suggestions for improvements. A table of their use pattern and their logged interactions with the App was used to ask follow up questions in regard to their specific use of the system.

\section{Results}

We analyzed the interview data using qualitative content analysis with partial transcription on audio recordings. Overall, we found that participants' expressed a general liking and interest in tracking their own smoking habits. We also found that no matter the age, all participants were keen on using a smartphone app in their smoking cessation.

In terms of the feedback provided by the app, over half of the users said that they learned something new about their smoking habit. One said, "I found out that I 
smoke the most at home and when passing time...I always thought that if I smoked, it was because I was stressed. So my justification to smoke is not really honest." All found it highly motivating to get a simple and informative picture of their smoking patterns. Those who were motivated to quit found the SMS reminders useful, while those not currently motivated to quit did not like being reminded.

Users preferred the convenience of receiving counseling on their phone rather than having to contact someone directly in order to get help. There was a correlation between users' motivation to quit and their acceptance of counseling messages. Users who took their quitting attempt seriously and wanted to change their habit, were very positive, saying, "It means a lot that it was written specifically to me, what I should do, and not what others should do". Those not wanting to quit yet said counseling made them feel pressured.

An interesting finding in respect to recording cigarettes resisted was that participants who were trying to quit said that registering resisted cigarettes and the situation made them crave a cigarette even more. Ironically, one participant rewarded himself with a cigarette for resisting one, "Good job, you have done well, smoke a cigarette for that." On the other hand, participants who were trying to stay quit found the Resisted button and graph very useful and liked that they could see their progress over time.

Most participants ( 9 out of 13 ) reported a positive change in their smoking habits after the three weeks of use. They had either reduced their consumption or QuittyLink had supported them in staying quit, "The App had a major impact; it has helped me to think about my consumption. It helped to postpone cigarettes." Those who did not change behavior reported that QuittyLink made them reflect more on their habits, "I have found out where my weakness lies. I think more about it every time I light a cigarette."

\section{Discussion and Conclusion}

The contribution of this work is the concept of giving smokers who are trying to quit personal counseling on smart phones, with insights about the potentials and challenges of this in smoking cessation. We have presented a prototype system, QuittyLink, designed to explore this concept, and studied it in use with people trying to quit. Contributing to the body of knowledge on technology-assisted smoking cessation, our results show that providing personalized counseling on smart phones, based on data about the individual user' $s$ smoking habits has promising potentials for helping people to achieve the goal of quitting, and staying quit.

Involving users in tracking of data about their smoking behavior helped make them aware of when, where and why they smoked thus supporting them in taking action to change that behavior if they wanted to. In addition to this, some reported that the personal counseling motivated them to make the effort to enter their data as they felt they were getting value from this. To our knowledge, this is a new approach to smoking cessation technology design. While computer-tailored content for smokers is not a new idea, using real-time experts to write counseling messages specifically for that individual, based on their actual smoking patterns and delivering it to them on smartphones is new - and it proved to be a motivator to reduce cigarette intake.

This study covered new ground in understanding opportunities for technology to contribute to providing supporting for individuals in their smoking cessation. To 
explore this we developed and evaluated QuittyLink, a mobile app, combining self-tracking and personal counseling. We found that QuittyLink promoted selfawareness and self-motivation and provided effective guidance and support for people wanting to quit smoking. People serious about quitting found the counseling messages extremely helpful. We found that the tracking offered by QuittyLink was considerably more accurate than smokers' own perceptions of their smoking habits, and that this allows people to develop more effective personal quitting strategies.

Finally, we found it interesting that self-tracking of "resisted" cigarettes can be counterproductive, leading smokers in an early quitting stage to actually smoke as a reward for resisting. We will use this and other findings from our study to redesign future iterations of QuittyLink that take account of smokers' different quitting stages.

\section{References}

[1] Abroms, L. C., Lee Westmaas, J., Bontemps-Jones, J., Ramani, R. and Mellerson, J. A content analysis of popular smartphone Apps for smoking cessation. Am. J. Prev. Med., 45, 6 (2013), 732-736.

[2] Chiu, M., Chang, S., Chang, Y., Chu, H., Chen, C. C., Hsiao, F. and Ko, J. Playful bottle. Proc. Ubicomp 2009, ACM Press, (2009), 185-194.

[3] Consolvo, S., Libby, R., Smith, I., Landay, J. A. McDonald, D. W., Toscos, T., Chen, M. Y., Froehlich, J., Harrison, B., Klasnja, P., Lamarca, A. and Legrand, L. Activity sensing in the wild. Proc. CHI 2008, ACM Press, (2008), 1797-1806.
[4] Free, C., Free, R., Whittaker, R., Knight, T., Abramsky, A. and Rodgers, I. G. Txt2stop: a pilot randomised controlled trial of mobile phone-based smoking cessation support. Tobacco control, 18, 2 (2009), 88-91.

[5] Paay, J., Kjeldskov, J., Brinthaparan, U., Lichon, L. Rasmussen, S., Srikandaraja, N., Smith, W., Wadley, G., Ploderer, B. Quitty: Using Technology to Persuade Smokers to Quit. Proc. NordiCHI 2014, ACM Press (2014).

[6] Ploderer, B., Smith, W., Pearce, J. and Borland, R. A Mobile App Offering Distractions and Tips to Cope With Cigarette Craving: A Qualitative Study. JMIR mHealth uHealth 2, 2 (2014), e23.

[7] Quit Victoria. Health risks of smoking,

http://www.quit.org.au/reasons-to-quit/health-risks-ofsmoking. Updated 2014.

[8] Schumann, A., John, U., Ulbricht, S., Ruge, J., Bischof, G. and Meyer, C. Computer-generated tailored feedback letters for smoking cessation: theoretical and empirical variability of tailoring. Int. J. Med. Inform., 77, 11 (2008), 715-722.

[9] Stead, L. F., Hartmann-Boyce, J., Perera, R. and Lancaster, T. Telephone counselling for smoking cessation. Cochrane Database Syst. Rev. 8 (2013), CD002850.

[10] Strecher, V. Computer-tailored smoking cessation materials: A review and discussion. Patient Education and Counseling, 36 (1999), 107-117.

11] World Health Organization. Tobacco. Fact Sheet 339

http://www.who.int/mediacentre/factsheets/fs339/en. Updated May 2014 